\title{
Chemical cross-linking controls in vitro fecal fermentation rate of high-amylose maize starches and regulates gut microbiota composition
}

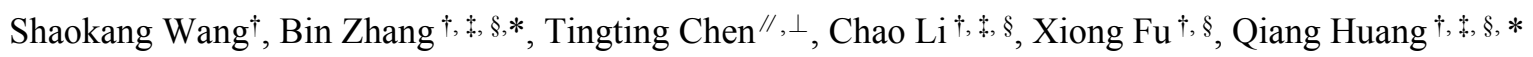

†School of Food Science and Engineering, National Research Center for Tropical Health Food, Guangdong Province

Key Laboratory for Green Processing of Natural Products and Product Safety, South China University of Technology,

Guangzhou 510640, China

†Sino-Singapore International Research Institute, Guangzhou 510555, China

§Overseas Expertise Introduction Center for Discipline Innovation of Food Nutrition and Human Health (111 Center), Guangzhou 510640, China

// Department of Biochemistry and Microbiology, Rutgers University, New Brunswick, NJ 08901-8525, United States

$\perp$ School of Food Science and Technology, Nanchang University, Nanchang 330047, China

* Corresponding authors

Tel.: +86 2087113845 ; fax: +86 2087113848 ;

Emails: zhangb@scut.edu.cn (B. Zhang); fechoh@scut.edu.cn (Q. Huang) 


\section{Supporting Information}

Table S1. IR Ratios and Increment of Gas and Short-chain Fatty Acid Production from 12 to 24 h for Crosslinked Starch Samples. (FOS, fructooligosaccharides; HAMS, high-amylose maize starch digesta; CL-5, CL-8, CL-12, crosslinked starches with low, medium and high crosslinking degree).

\begin{tabular}{ccccccc}
\hline Samples & $\begin{array}{c}1047 / 1022 \\
\mathrm{~cm}^{-1}\end{array}$ & $\begin{array}{c}\Delta \text { Gas } \\
(\%)\end{array}$ & $\begin{array}{c}\Delta \text { Acetate } \\
(\%)\end{array}$ & $\begin{array}{c}\Delta \text { Propionate } \\
(\%)\end{array}$ & $\begin{array}{c}\Delta \text { Butyrate } \\
(\%)\end{array}$ & $\begin{array}{c}\Delta \text { Total SCFA } \\
(\%)\end{array}$ \\
\hline FOS & --- & 4.00 & 12.65 & 1.30 & 0.42 & 9.63 \\
HAMS & 0.9695 & 6.30 & 16.25 & 1.92 & 0.35 & 11.36 \\
CL-5 & 0.9233 & 23.80 & 39.03 & 8.82 & 8.96 & 31.45 \\
CL-8 & 0.8122 & 34.50 & 48.65 & 19.75 & 15.84 & 35.21 \\
CL-12 & 0.8078 & 50.00 & 32.08 & 14.11 & 9.68 & 24.65 \\
\hline
\end{tabular}


Figure S1. Phylum level changes in microbiota composition of crosslinked after the in vitro fermentation for 0, 4, 8 , 12 and 24 h. (FOS, fructooligosaccharides; HAMS-D, high-amylose maize starch digesta; CL-5-D, CL-8-D, CL12-D, crosslinked starches with low, medium and high crosslinking degree subjected to in vitro small intestine digestion).

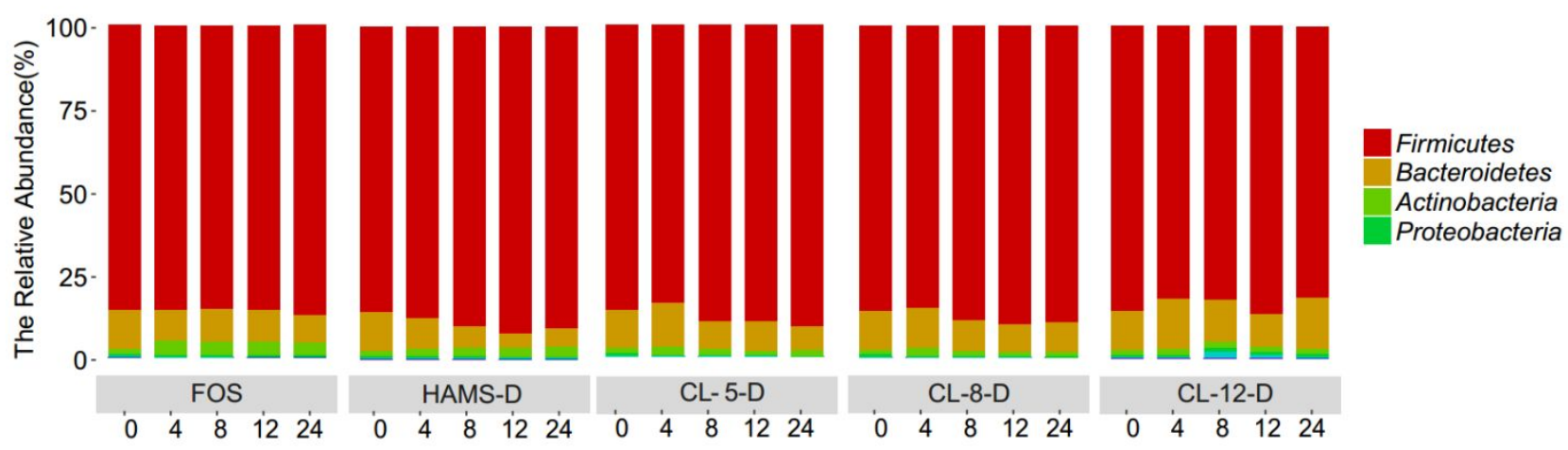

